\title{
Categorías de género: consecuencias cognitivas sobre la identidad
}

\author{
Miguel C. Moya Morales \\ Universidad de Granada
}

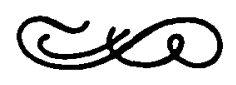

Resumen

En este artículo se presentan algunas reflexiones en tomo a los aspectos cognitivos de la identidad de género, ilustrándolas con datos procedentes de investigaciones realizadas en nuestro país. En primer lugar se aborda la cuestión de la importancia que la pertenencia a las categorias de género tiene para bombres y mujeres, asi como la conciencia de esta pertenencia. Posteriormente se consideran las autopercepciones que bombres y mujeres desamollan como consecuencia de sus pertenencias categoriales de género, particularmente a la luz de los índices de masculinidad, feminidad y androginia encontrados en muestras españolas. Por último, se revisan algunos factores que influyen en la autopercepción en téminos de las categorias de género: grado de identificación con el endogrupo de género, status de la categoría, características de la situación y tono evaluativo de los atributos estereotípicos.

Palabras clave: Categorías de género; Identidad de género.

\section{Gender categories: Cognitive Consequences for Identity}

\section{Abstract}

We present some ideas about cognitive aspects fo gender identity and illustrate these thoughts with data from some studies carried out in our country. First, we consider the issue of the importance and awareness of gender categories for males and females. Second, we consider the specific self-perceptions of men and women, taking into account the indices of masculinity, feminity and androgyny found in Spanish samples. Finally, we review some factors that influence self-penceptions in terms of gender categories: strength of gender ingroup identification, category's status, situational characteristics and evaluative tone of stereotypical attributes.

Key words: Gender categories; Gender identity.

Dirección del autor: Facultad de Filosofía y Letras, Departamento de Psicología Social. Universidad de Granada 18071. Granada. 


\section{INTRODUCCION}

Existen numerosos criterios en virtud de los cuales podemos ser clasificados. Uno de ellos es el sexo. Las distinciones basadas en el sexo biológico representan el criterio de categorización social más utilizado, tanto a lo largo del tiempo como a través de diferentes culturas (Goffman, 1977). Es, además, quizás el único sistema de categorización que es universal, en el sentido de que afecta a todos los seres humanos. En los niños y niñas el sexo es una de las primeras categorías cognitivas. Con poco más de dos años, niños y niñas son capaces de atribuir correctamente la etiquea sexual y conocen lo esencial de los estereotipos de género (Hurtig y Pichevin, 1985).

El término «sexo» tiene poderosas connotaciones biológicas y subraya fundamentalmente la existencia de diferencias anatómicas. Por esta razón, en ciencias sociales cada vez es más frecuente el uso del término «género», que posee un carácter marcadamente sociológico, y sirve para designar un esquema utilizado para la categorización social de los individuos (Sherif, 1982; Moya, 1985). Todo esquema de género reconoce la diferenciación biológica, pero a la vez crea diferenciaciones sociales. En nuestra sociedad, y en esto no es desde luego la única, la categorización hombre-mujer, o masculino-femenino, llega hasta los más recónditos lugares e impregna los procesos más insospechados. Desde que nacemos aprendemos una serie de definiciones culturales de la masculinidad y la feminidad que comprenden un diverso y amplio conjunto de asociaciones ligadas al sexo: anatomía, función reproductora, división del trabajo, atributos de personalidad, conductas de rol, apariencia física, etc., llegando incluso a rasgos que están metafóricamente o remotamente ligados al sexo, como las formas geométricas o los colores.

La categorización basada en el género, como toda categorización, tiene la función primordial de ayudar, simplificando, en la percepción de la compleja realidad social. Además, categorizar nos ayuda en la realización de inferencias, esto es, a predecir cómo será el comportamiento de los integrantes de cierta categoría y, en consecuencia, a prever cómo ha de ser nuestro propio comportamiento. Imaginémonos que un amigo residente en una ciudad lejana nos pide que alojemos en nuestra casa por unos días a un familiar suyo. Nuestras previsiones y comportamiento en relación con la acogida variarán enormemente de saber que esa «persona» es un «hombre» a saber que es una «mujer». Como indica Sherif (1982), cuando se clasifica a las personas dentro de categorías, invariablemente se forman un conjunto de normas sociales que sirven para evaluar a sus integrantes y se desarrollan reglas acerca de cómo han de relacionarse los miembros de diferentes categorías.

Además, dado que la categorización sexual o de género sólo comprende dos categorías (aunque existen ciertas categorías especiales para designar algunos casos ambiguos, por ejemplo, eunucos o transexuales), la relación con una de estas categorías lleva casi inexorablemente implícita la relación con la otra. De esta manera, la identidad de género se refiere a una relación psicológica del individuo con las categorías de género de una sociedad; no sólo con la categoría a la que pertenece, sino también con aquellas categorías relacionadas. En nuestra sociedad resulta imposible pensar en lo que significa «ser hombre» sin que a la vez se esté pensando en lo que significa «ser mujer». Cuando se dice «los hombres no lloran» se está diciendo al mismo tiempo que «las mujeres sí lo hacen». 
Una de las características más universales del sistema de categorización sexual es su carácter asimétrico, esto es, las diferencias existentes entre los miembros de ambas categorías de género en los accesos a recursos, poder, prestigio y status en general. Son las mujeres quienes constituyen el grupo de status inferior y los hombres el de status superior en todas las sociedades conocidas, al menos en aquellos asuntos que afectan de manera más clara a la supervivencia de la comunidad (Friedl, 1975).

Ahora bien, una vez mostrada la existencia de la categorización en función de género y establecidas algunas características generales de este sistema de categorías, la pregunta que como psicólogos sociales nos hacemos es: ¿cómo esta realidad «social» influye en la psicología de los individuos?

Según la Teoría de la Identidad Social (desde ahora, TIS) (Tajfel, 1984; Tajfel y Turner, 1979), los grupos o categorías sociales a los que pertenecemos influyen en nuestra identidad social, o mejor dicho, forman parte de ella. La identidad social es «el conocimiento por parte del individuo de que pertenece a ciertos grupos sociales junto con la significación emocional y valorativa de esa pertenencia para él» (Tajfel, 1972, p. 292). Así pues, la categorización de género no sólo se utiliza para la percepción de la realidad externa, sino también para la percepción de nosotros mismos. En la definición previa puede observarse cómo hay tres componentes que forman parte de la identidad social. El primero, cognitivo, designa el conocimiento que un individuo tiene de pertenecer al grupo, o conciencia de pertenencia; el segundo, evaluativo, se refiere al hecho de que generalmente esa conciencia de pertenencia está impregnada de algunas connotaciones de valor, positivas o negativas; por último, el componente emocional, indica que los dos componentes anteriores pueden ir acompañados de emociones - amor, odio, agrado, etc.- hacia el propio grupo o hacia grupos que mantienen relaciones con él. El presente trabajo se centra en el primero de los componentes mencionados, las consecuencias cognitivas de pertenecer a una categoría género. Estas consecuencias son, básicamente, la conciencia de pertenencia, la importancia que tiene esa pertenencia dentro de la identidad social global del individuo y los contenidos concretos que se derivan de la pertenencia categorial.

No obstante, resulta muy difícil considerar el aspecto cognitivo sin hacer referencia a los otros aspectos, especialmente al evaluativo. Asumiendo que las personas buscamos tener una identidad, o un concepto de nosotros mismos, positivo, y que la identidad social deriva de las pertenencias grupales, resulta pues necesario que los grupos a los que pertenecemos mantengan una «distintividad» positiva respecto a los otros grupos, de manera que la identidad que nos proporciona sea positiva. De esta manera, a través de la comparación social, identidad y relaciones intergrupales aparecen estrechamente vinculadas entre sí. Sin embargo, las consecuencias intergrupales de la pertenencia a las categorías de género no serán tratadas, por razones de espacio, en este trabajo.

\section{CONCIENCIA E IMPORTANCIA DE LA PERTENENCIA A CATEGORIAS DE GENERO}

Según algunos autores, la pertenencia a la categoría de género repercute con mayor intensidad en las mujeres que en los hombres (Lorenzi-Cioldi, 1988). Este último autor considera que la pertenencia categorial de los grupos domina- 
dos es más saliente que la de los grupos dominantes, de ahí que las mujeres tiendan a verse más en términos de sus características grupales y los hombres más en términos de sus características personales. En ocasiones este fenómeno puede manifestarse de forma sutil. Por ejemplo, McGuire et al. (1979), en un estudio realizado con cerca de 600 adolescentes, encontraron que no había diferencias entre ambos sexos en las respuestas a la pregunta «Díme quién eres tú». En cambio, cuando la pregunta fue «Dime quién no eres tú», sí que aparecieron diferencias, siendo las chicas quienes con mayor frecuencia indicaban su grupo de género de no pertenencia. Sin embargo, otras investigaciones muestran un patrón de resultados opuesto. Mulford y Salisbury (1964), estudiando las respuestas de 1.213 personas representativas de la población adulta de Iowa al TST (Twenty Statement Test), encontraron que los hombres mencionaron más que las mujeres su identidad de género $-25 \%$ versus $10 \%(\mathrm{p}<.05)-$ Estos resultados no concuerdan con los que M. Kuhn (1960) había encontrado en una muestra de estudiantes universitarios utilizando el mismo instrumento. Los resultados de Mulford y Salisbury parecen, por otra parte, bastante lógicos: la expresión de la pertenencia grupal debería ser mayor en el grupo dominante, esto es, en aquel que proporciona a sus miembros una identidad social positiva.

Seguidamente expondremos los datos de dos investigaciones en las cuales se abordó la cuestión de la importancia y matices de la identidad de género de varones y mujeres. Entre los objetivos de estas investigaciones estaban examinar si existían o no componentes diferenciados dentro de la identidad de género, considerar la relación entre estos componentes y ver si ambos fenómenos se dan exactamente igual en hombres y mujeres. En la primera investigación (Moya, 1991), participaron 149 estudiantes de un centro de Formación Profesional (32 varones y 117 mujeres, y la edad promedio fue de 21 años). El diagnóstico de la identificación de los individuos con su endogrupo sexual se realizó utilizando una versión modificada de la «Group Identification Scale» (Brown et al., 1986) (ver Tabla II).

Analizando los datos de todos los participantes conjuntamente, mediante un análisis factorial de componentes principales - rotación varimax-, se obtuvieron 3 factores que explicaban el $54 \%$ de la varianza (el coeficiente Alpha de Cronbach fue .73). El primer factor agrupaba a los ítems con un claro componente emocional y evaluativo negativo y explicaba el $29,8 \%$ de la varianza: «me molesta reconocer que soy $\mathrm{v} / \mathrm{m} \gg(.73)$, «intento ocultar mi pertenencia al grupo de los $\mathrm{v} / \mathrm{m}$ » (.68), «me siento limitado por el grupo de $\mathrm{v} / \mathrm{m}$ » (.67), «me avegüenzo de pertenecer al grupo de los $\mathrm{v} / \mathrm{m} »(.65)$ y «me siento incómodo cuando estoy con otros $\mathrm{v} / \mathrm{m»}(.59)$. Los ítems con altas saturaciones en el segundo factor tenían una clara referencia cognitiva (que explicaban conjuntamente el $18,7 \%$ de la varianza): «me identifico con los $\mathrm{v} / \mathrm{m}$ » (.77), «siento fuertes vínculos con los $\mathrm{v} / \mathrm{m}$ ( (.76) y «me considero miembro del grupo de los $\mathrm{v} / \mathrm{m}$ ( (.49). Por último, el tercer factor (que explicaba el $10,9 \%$ de la varianza) sólo agrupaba a dos ítems de carácter positivo: «me compenetro bien con el grupo de los v/m» (.66) y «me alegro de ser $\mathrm{v} / \mathrm{m} \gg(.62)$.

Se obtuvieron las correlaciones entre sí de los tres factores y de la puntuación total en la escala (Tabla I).

Considerando a todos los sujetos, puede apreciarse cómo en general no existen unas correlaciones altas entre los tres componentes obtenidos en la escala de identificación con el endogrupo de género, aunque son significativas en dos de los casos. El componente cognitivo y el evaluativo-negativo correlacionan 


\section{5}

TABLA I

Correlaciones dentro de la escala de identificación con el endogrupo de género (Moya, 1991).

\begin{tabular}{cccccccccc}
\hline & \multicolumn{2}{c}{$\begin{array}{c}\text { todos }(\mathrm{n}=149) \\
\end{array}$} & \multicolumn{3}{c}{ varones $(\mathrm{n}=32)$} & \multicolumn{3}{c}{ mujeres $(\mathrm{n}=117)$} \\
& 3 & total & 2 & 3 & total & 2 & 3 & total \\
\hline 1 & $-.2^{\star}$ & -.11 & $-.79^{\star \star \star}$ & -.2 & -.26 & $-.88^{\star \star \star}$ & .10 & .12 & $-.73^{\star \star \star}$ \\
2 & & $.38^{\star \star \star}$ & $.67^{\star \star \star}$ & & .009 & $.53^{\star \star}$ & & -.006 & $.45^{\star \star \star}$ \\
3 & & & $.55^{\star \star \star}$ & & & $.46^{\star \star}$ & & & $.59^{\star \star \star}$
\end{tabular}

${ }^{*} \mathrm{p}<.05,{ }^{* * *} \mathrm{p}<.001$

negativamente (-.2), mientras que el cognitivo y el evaluativo-positivo presentan un índice de correlación mayor y de signo positivo (.38). Esto indica que cuando el grupo de género proporciona una identidad social negativa hay paralelamente una baja identificación cognitiva con él, mientras que si la identidad es positiva, aumenta esta identificación cognitiva. No obstante, al considerar las correlaciones por separado de hombres y mujeres participantes, encontramos que esta relación inversa entre identidad negativa e identidad cognitiva sólo se da en el caso de los hombres; en las mujeres la correlación es muy débil y de signo positivo. De esta manera, tal y como indica la TIS (Tajfel y Turner, 1979), cuando un grupo no proporciona identidad social positiva, una de las posibilidades que tienen sus miembros consiste en disminuir la vinculación con dicho grupo. Lo llamativo del resultado es que este proceso sólo se dé en los varones. Esta estrategia, denominada por Tajfel y Turner «movilidad individual», implica que la situación del endogrupo como un todo continúe igual y que los individuos adopten una alternativa individualista. En cambio, las mujeres parecen tener un sentido de «grupalidad» mayor, pues no utilizan esta estrategia individualista. Además, curiosamente, en los hombres el componente positivo y el negativo de la identidad correlaciona negativamente - como cabría esperar-, mientras que en las mujeres jcorrelaciona positivamente! De esta manera, los resultados sugieren cómo las mujeres parecen no tener más remedio que aceptar su pertenencia grupal, con los aspectos tanto positivos como negativos que conlleva. La puntuación total obtenida en la escala fue muy similar para hombres y mujeres participantes (5.81 y 5.88, respectivamente). En los varones, tiene una mayor correlación con la identificación global el componente cognitivo que el evaluativo-positivo, mientras que en las mujeres es al revés.

En la segunda investigación (Moya, 1990), un análisis similar al que acabamos de exponer arrojó resultados parecidos. En este caso las personas que participaron en el estudio fueron 224 estudiantes de Bachillerato (101 varones y 123 mujeres). Aquí sí aparecieron diferencias significativas entre hombres y mujeres en la puntuación total de identificación con el endogrupo de género, siendo mayor esta puntuación en las mujeres (5.9) que en los hombres $(5.73)[\mathrm{F}(1,208)=$ $4.93, \mathrm{p}<.03$ ]. En cambio, cuando se consideran cada uno de los ítems, como puede observarse en la Tabla II, sólo en dos de ellos aparecieron diferencias significativas: las mujeres consideraban que su endogrupo de género era más importante y los varones se consideraban miembros de su endogrupo en mayor medida. 


\section{6}

TABLA II

Puntuaciones de varones y mujeres en los items de la escala de identificación con el endogrupo de género.

\begin{tabular}{lccc}
\hline Item & $\begin{array}{c}\text { Varones } \\
(\mathrm{n}=102)\end{array}$ & $\begin{array}{c}\text { Mujeres } \\
(\mathrm{n}=122)\end{array}$ & $\mathrm{F}$ \\
& $(\mathrm{n}=102$ \\
\hline
\end{tabular}

Considero que los $\mathrm{v} / \mathrm{m}$ son importantes

$\begin{array}{lcc}4.80 & 5.87 & 36.33^{\star * *} \\ 5.38 & 5.55 & .40 \\ 3.72 & 4.26 & 5.7 \\ 6.35 & 6.46 & 2.46 \\ 6.23 & 5.79 & 5.42^{\star} \\ 1.40 & 1.43 & .03 \\ 1.69 & 1.45 & 1.01 \\ 2.34 & 2.48 & .99 \\ 1.37 & 1.34 & .02 \\ 1.96 & 1.61 & 1.54 \\ 4.95 & 5.16 & .06\end{array}$

$\mathrm{Me}$ identifico con los $\mathrm{v} / \mathrm{m}$

Siento fuertes vínculos con los $\mathrm{v} / \mathrm{m}$

$\mathrm{Me}$ alegro de ser $\mathrm{v} / \mathrm{m}$

Me considero miembro del grupo de los $\mathrm{v} / \mathrm{m}$

Me avergüenzo de pertenecer al grupo de los $\mathrm{v} / \mathrm{m}$

Intento ocultar mi pertenencia al grupo de los $\mathrm{v} / \mathrm{m}$

Me siento limitado por el grupo de los $\mathrm{v} / \mathrm{m}$

$\mathrm{Me}$ molesta reconocer que soy $\mathrm{v} / \mathrm{m}$

Me siento incómodo cuando estoy con otros $\mathrm{v} / \mathrm{m}$

Me compenetro bien con los $\mathrm{v} / \mathrm{m}$

4.95

1.61

.06

$\star \star \star \mathrm{p}<.001 ;{ }^{\star} \mathrm{p}<.01$

De los resultados de las anteriores investigaciones podemos deducir que la indentificación con el grupo de género es bastante importante tanto para los hombres como para las mujeres. En una escala de 7 puntos, los hombres del primer estudio obtienen una media de 5.81 y los del segundo estudio de 5.73, y las mujeres del primer estudio de 5.88 y las del segundo de 5.9. No aparece un patrón claro de respuesta que indique que la fuerza de la identificación es mayor en un sexo que en otro. En cambio sí que aparecen diferencias entre hombres y mujeres cuando se consideran los aspectos evaluativos y cognitivos de la identificación con el endogrupo.

\section{CONTENIDOS DERIVADOS DE LA PERTENENCIA A LA CATEGORIA DE GENERO}

¿Cómo se autoperciben los varones y las mujeres? A continuación intentaremos responder a esta pregunta con algunos datos obtenidos en nuestro país. Posteriormente mostraremos cómo estas autopercepciones no son estáticas, sino que experimentan cambios y fluctuaciones dependiendo de una serie de factores, algunos de los cuales serán referidos.

El método generalmente utilizado para estudiar la autopercepción según los estereotipos de género consiste en presentarle a los sujetos una serie de características - que previamente han aparecido asociadas de forma diferente a hombres y a mujeres - y pedirles que se autocalifiquen sobre tales atributos. El instrumento más popular en esta área es el Bem Sex Role Inventory (Bem, 1974). Este cuestionario consta de 60 ítems (20 masculinos, 20 femeninos y 20 de deseabilidad social) y a los sujetos se les pide que apliquen cada ítem a sí mismos, utilizando una escala de 7 puntos (1, nunca o casi nunca; 7 , siempre o casi siempre). En una investigación realizada con estudiantes de Psicología utilizando este instrumento los resultados mostraron que las autodescripciones de varones y mujeres eran muy similares. De 60 ítems, sólo en 5 hay diferencias significativas: las mujeres se autodescribían como más enérgicas, femeninas y alegres, mientras que los hombres lo hacían como más masculinos y cálidos. 
En otra investigación (Moya, 1990) se utilizó un instrumento de elaboración propia y también se les pidió a los sujetos (en este caso estudiantes de BUP) que se aplicaran a sí mismos cada uno de los ítems utilizando una escala de 7 puntos (1, nunca o casi nunca; 7, siempre o casi siempre). En este caso sí aparecieron algunas diferencias entre los varones y las mujeres. Los hombres se autodescribían como más altos, ambiciosos, anchos de hombros, atléticos, fuertes y violentos; y las mujeres como más capaces de captar los sentimientos de los demás, cariñosas, emocionales, sensibles, soñadoras, de habla suave y con mucho tacto en su relaciones con los demás. En este caso la autodescripción de varones y mujeres coincide en líneas generales con los estereotipos tradicionales masculinos y femeninos (Moya y Pérez, 1990), si bien las diferencias se dan en muchos menos ítems de los habitualmente considerados típicos del hombre y de la mujer.

Moya y Gómez (1991), en otra investigación en la que se utilizó un instrumento similar, realizada con una muestra de estudiantes de Psicología de las universidades de Granada, Sevilla, Valencia, País Vasco y La Laguna, obtuvieron los resultados que aparecen en la Tabla III.

En este caso, sí aparecieron diferencias entre los varones y mujeres en más ítems. Sólo no hubo diferencias significativas en los siguientes: Atrevido/a, Mu-

\section{TABLA III}

Puntuaciones medias ${ }^{1} y$ desviaciones típicas obtenidas por los varones $y$ mujeres estudiantes de Psicología en diferentes atributos.

\begin{tabular}{|c|c|c|c|c|c|}
\hline \multirow[t]{2}{*}{ Item } & \multicolumn{2}{|c|}{$\begin{array}{c}\text { Varones } \\
(\mathbf{n}=170)\end{array}$} & \multicolumn{2}{|c|}{$\begin{array}{l}\text { Mujeres } \\
(n=468)\end{array}$} & \\
\hline & Media & S.D. & Media & S.D. & \\
\hline Agresivo/a & 2.60 & 1.33 & 2.35 & 1.18 & $5.51^{*}$ \\
\hline Amable & 5.13 & 1.07 & 5.31 & .86 & $4.63^{*}$ \\
\hline Ambicioso/a & 4.37 & 1.51 & 3.95 & 1.51 & $9.89^{\star \star}$ \\
\hline Atlético/a & 3.79 & 1.76 & 2.62 & 1.50 & $67.00^{\star \star \star}$ \\
\hline Atrevido/a & 3.91 & 1.55 & 3.68 & 1.47 & 3.13 \\
\hline Cariñoso/a & 4.85 & 1.34 & 5.26 & 1.11 & $14.02^{* * *}$ \\
\hline $\begin{array}{l}\text { Capaz de captar los sentimientos de } \\
\text { los demás }\end{array}$ & 5.10 & 1.12 & 5.40 & .99 & $9.99^{\star *}$ \\
\hline Mucho tacto en sus relaciones con & & & & & \\
\hline $\begin{array}{l}\text { los demás } \\
\text { Competitivo/a }\end{array}$ & $\begin{array}{l}4.98 \\
4.01\end{array}$ & $\begin{array}{l}1.23 \\
1.56\end{array}$ & $\begin{array}{l}5.11 \\
3.49\end{array}$ & $\begin{array}{l}1.22 \\
1.40\end{array}$ & $\begin{array}{c}1.43 \\
17.09^{* * *}\end{array}$ \\
\hline Defiende sus propias ideas & 5.55 & 1.18 & 5.49 & 1.23 & .29 \\
\hline Dominante & 3.70 & 1.61 & 3.62 & 1.61 & .39 \\
\hline Emocional & 4.87 & 1.56 & 5.56 & 1.28 & $31.74 * * *$ \\
\hline Femenino/a & 2.32 & 1.39 & 5.54 & 1.32 & $691.98^{* \star *}$ \\
\hline De habla suave & 3.79 & 1.63 & 4.41 & 1.50 & $21.73^{\star * \star}$ \\
\hline Independiente & 5.03 & 1.36 & 4.76 & 1.42 & $4.25^{\star}$ \\
\hline Individualista & 4.37 & 1.78 & 3.62 & 1.63 & $24.53^{* * *}$ \\
\hline Masculino/a & 5.42 & 1.24 & 1.88 & 1.19 & $1047.8^{\star \star \star}$ \\
\hline Meticuloso/a & 4.15 & 1.60 & 3.95 & 1.68 & 1.49 \\
\hline Paciente & 4.43 & 1.57 & 4.39 & 1.52 & .07 \\
\hline Sensible & 5.44 & 1.27 & 5.96 & .99 & $28.74^{* * *}$ \\
\hline Soñador/a & 5.26 & 1.43 & 5.56 & 1.36 & 6.29 \\
\hline Violento/a & 2.29 & 1.29 & 1.96 & 1.08 & 10.97 \\
\hline
\end{tabular}

\footnotetext{
${ }^{1}$ Se utilizó una escala de 7 puntos (de 1 - nunca o casi nunca- a 7 -siempre o casi siempre-).
} 


\section{8}

cho tacto en sus relaciones con los demás, Defiende sus propias ideas, Dominante, Meticuloso/a y Paciente. Las mujeres puntúan más alto que los varones en Amable, Cariñoso/a, Capaz de captar los sentimientos de los demás, Emocional, Femenino/a, De habla suave, Sensible y Soñador/a. Los varones puntúan significativamente más alto que las mujeres en Agresivo/a, Ambicioso/a, Atlético/a, Competitivo/a, Independiente, Individualista, Masculino/a y Violento/a. Los hombres puntúan más alto que las mujeres en los ítems masculinos y éstas obtienen puntuaciones mayores que aquéllos en los femeninos, sin embargo, aparece una interacción: las puntuaciones en los ítems masculinos tienden a ser menores que en los femeninos, o dicho de otra manera, tanto las mujeres como los hombres puntúan más alto en los ítems femeninos que en los masculinos, si bien las diferencias entre las puntuaciones obtenidas en ambos conjuntos de ítems son mayores en el caso de las mujeres. Estos resultados pueden observarse en la Figura 1.

FIGURA 1

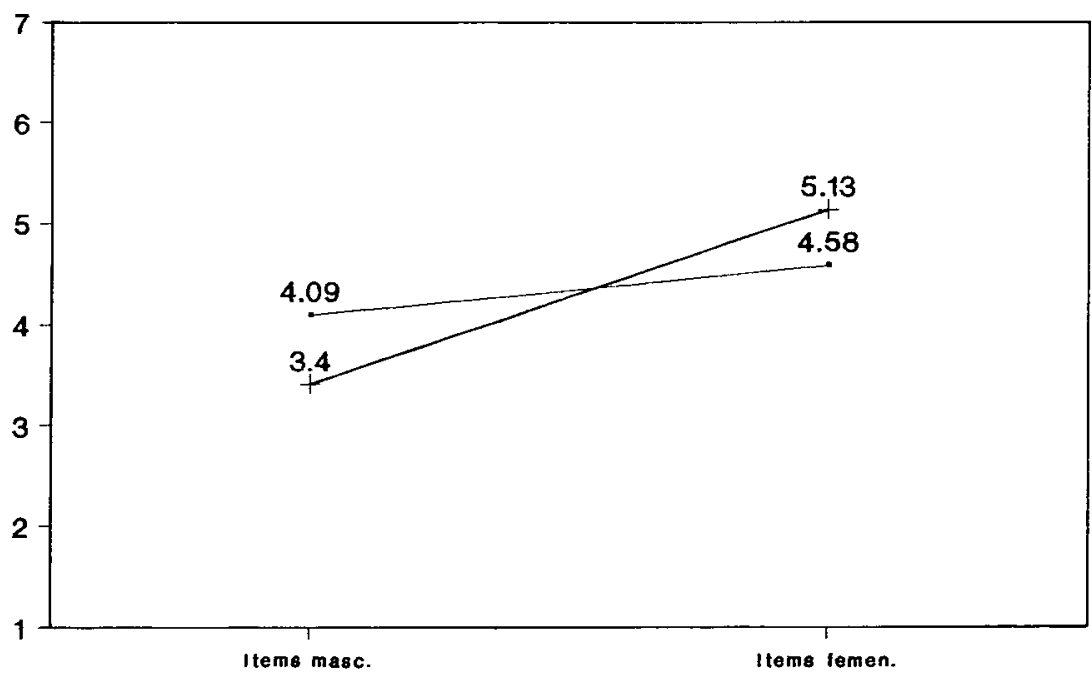

-Masc.items + Fem. items

Autocalificación sexo $\times$ tipo ítem

Otra forma de aproximarse al estudio de la autoestereotipia consiste en considerar las puntuaciones globales en los ítems masculinos y femeninos. Según la corriente predominante en este área, masculinidad y feminidad son dos dimensiones independientes, esto es, que cualquier persona puede puntuar alto en las dos dimensiones, o bajo en las dos, o alto en una y bajo en otra. De esta manera surgen cuatro grandes grupos de individuos en función de su orientación del rol sexual (independientemente de su sexo biológico): masculinos (altos en masculinidad y bajos en feminidad), femeninos (altos en feminidad y bajos en masculinidad), andróginos (altos en masculinidad y feminidad) e indiferenciados (bajos en masculinidad y feminidad). Los resultados de gran parte 
de las investigaciones han mostrado que las mujeres son superiores en femini$\mathrm{dad}$, los hombres en masculinidad y respecto a la androginia no hay resultados consistentes. Algunos datos indican que el porcentaje de andróginos es mayor en las mujeres (Bem, 1974), lo que unido al resultado de que suele haber más mujeres masculinas que hombres femeninos (Basow, 1980) se ha interpretado como un apoyo a la idea de que la tipificación sexual del varón es más rígida que la de la mujer. Otros datos, en cambio, sugieren que el porcentaje de andróginos es mayor entre los varones (Heilbrum, 1981).

En la siguiente tabla pueden observarse los resultados de algunas investigaciones realizadas en nuestro país que han utilizado el BSRI -Bem Sex Role Inventory (Bem, 1974)-. En todas ellas se utilizó el criterio habitualmente empleado en las investigaciones sobre orientación del rol sexual para crear diversas categorías de sujetos en función de esta orientación. Dicho criterio consiste en considerar en cada sujeto su puntuación en masculinidad y feminidad (ítems masculinos y femeninos, respectivamente) y comparar cada una de estas puntuaciones con la mediana del grupo. También aparecen los resultados de dos investigaciones en las que se utilizó un instrumento diferente (Moya, 1990, 1991). En todos los casos, se considera que un individuo es «masculino» cuando puntúa por encima de la mediana en los ítems masculinos y por debajo en los femeninos; que es «femenino», cuando el patrón de respuesta es el inverso; que es andrógino cuando puntúa por encima de la mediana tanto en los ítems masculinos como en los femeninos; y que es indiferenciado cuando puntúa por debajo de la mediana en los dos grupos de ítems.

\section{TABLA IV}

Porcentajes de individuos masculinos, femeninos, andróginos e indiferenciados encontrados en varias investigaciones realizadas en España.

\begin{tabular}{ccccccc}
\hline Autor & Sexo & $\mathrm{n}$ & Masc. & Femen. & Andróg. & Indif. \\
\hline $\begin{array}{c}\text { Fernández (1982) } \\
\text { (BSRI) }\end{array}$ & $\mathrm{V}$ & 46 & 28.26 & 13.04 & 28.26 & 30.43 \\
P & 68 & 19.12 & 29.42 & 27.94 & 23.53 \\
$\begin{array}{c}\text { Páez et al. (1989) } \\
\text { (BSRI) }\end{array}$ & $\mathrm{V}$ & 90 & 37 & 14 & 31 & 17 \\
& $\mathrm{M}$ & 95 & 13 & 28 & 20 & 39 \\
$\begin{array}{c}\text { Echebarría (1990) } \\
\text { (BSRI) }\end{array}$ & $\mathrm{V}$ & 90 & 36.7 & 11.1 & 27.8 & 24.4 \\
Moya (1987) & $\mathrm{V}$ & 107 & 6.5 & 31.8 & 31.8 & 29.9 \\
(BSRI) & $\mathrm{M}$ & 114 & 21.93 & 31.58 & 17.54 & 21.05 \\
Moya (1990) & $\mathrm{V}$ & 101 & 36.2 & 12.8 & 21.3 & 29.8 \\
& $\mathrm{M}$ & 123 & 12.6 & 39.6 & 28.8 & 18.9 \\
Moya (1991) & $\mathrm{V}$ & 32 & 25 & 28 & 22 & 25 \\
& $\mathrm{M}$ & 117 & 26 & 20.5 & 27 & 26.5 \\
\hline
\end{tabular}

En resumen, las anteriores investigaciones indican que en ciertos casos hay más mujeres masculinas que hombres femeninos (Fernández, 1982; Moya, 1987; Moya y Gómez, 1991), en otro ocurre al revés (Echebarría) y en otros apenas 
hay diferencias (Páez et al.; Moya, 1990, 1991). En dos investigaciones el porcentaje de sujetos andróginos es bastante mayor entre los hombres que entre las mujeres (Páez et al., 1989; Moya, 1987). En la investigación de Fernández (1982) apenas hay diferencias y en tres estudios hay un predominio de mujeres andróginas (Echebarría, 1990; Moya, 1990, 1991).

Si consideramos la puntuación únicamente en los ítems masculinos y femeninos (sin comparar con el grupo o con la puntuación en otros ítems), entonces los resultados varían. En dos de las investigaciones realizadas por nosotros (Moya, 1990, 1991), la puntuación es mayor en los ítems femeninos que en los masculinos (4.64 vs. 4.18 en la primera investigación y 4.42 vs. 4.11 en la segunda). Este patrón de resultados es consecuencia sobre todo de la tendencia de respuesta de las mujeres ( 4.71 vs. 3.94 en la primera investigación y 4.75 vs. 3.85 en la segunda). En los varones, la puntuación es ligeramente superior en los ítems masculinos en la primera investigación (4.13 vs. 4.29) y apenas hay diferencias en la segunda ( 4.53 vs. 4.52 ).

En general, de estas investigaciones se desprende que si bien los hombres y las mujeres se autoperciben en términos diferentes y coincidentes en líneas generales con los estereotipos de género tradicionales, no lo hacen de forma tan generalizada y drástica como con frecuencia se ha supuesto. En primer lugar, hay muchos atributos que los hombres y las mujeres se autoadjudicaban en igual medida; en segundo lugar, hay casos en los que los hombres se autoadjudican más que las mujeres atributos tradicionalmente femeninos (e.g. «cálido») y éstas más que aquéllos atributos masculinos (e.g. «enérgico»). En tercer lugar, las diferencias encontradas en los resultados de los diversos estudios sugieren la importancia de otras categorizaciones, aparte de la del género, en la autoestereotipia. Por ejemplo, tanto las mujeres estudiantes de BUP como las de Psicología se consideran bastante «capaces de captar los sentimientos de los demás» y apenas hay diferencias entre ellas (5.50 vs. 5.40); en cambio, los varones estudiantes de Psicología puntúan mucho más alto en ese ítem (5.10) que los estudiantes de BUP (4.66).

Por último, no queda nada claro el patrón de autoadjudicación de rasgos masculinos y femeninos en hombres y mujeres. Todo parece depender del criterio utilizado. Si nos fijamos en las puntuaciones absolutas en los ítems masculinos y femeninos, parece ser que tanto hombres como mujeres se aproximan a los rasgos femeninos. En cambio, si la puntuación de hombres y mujeres obtenida en los ítems masculinos y femeninos se considera conjuntamente y se relativiza en función de la puntuación obtenida por el grupo (que es lo que se hace en el BSRI e instrumentos similares), entonces, aparece cierta tendencia mayor en las mujeres que en los hombres a autoadjudicarse características del sexo opuesto y no del propio. Si lo que se considera es el porcentaje de varones y mujeres que se autoadscriben tanto características masculinas como femeninas, entonces las investigaciones se dividen casi al cincuenta por ciento entre aquellas que muestran que esta tendencia es mayor en los hombres y aquellas que la han encontrado en las mujeres.

\section{ALGUNOS FACTORES QUE INFLUYEN EN LAS AUTOPERCEPCIONES}

Hasta aquí hemos visto cómo hombres y mujeres se autoperciben en términos estereotípicos. Dicha autopercepción se ha diagnosticado de una forma ge- 


\section{1}

neral, prescindiendo de las situaciones concretas en las que las personas nos encontramos diariamente. Sin embargo, hay razones suficientes para pensar que esta autoconcepción varía según las circunstancias o las características de la situación. Dion (1975), por ejemplo, relata cómo cuando las mujeres son discriminadas por el hombre, sólo aquellas mujeres que se dan cuenta del prejuicio tienden a aumentar la visión de sí mismas en concordancia con el estereotipo femenino.

Según la teoría de la Identidad Social, cuando las personas en una interacción social actúan en función de su pertenencia categorial (es decir, las relaciones se sitúan en el extremo intergrupal del continuo interpersonal-intergrupal), mostrarán una mayor uniformidad en su conducta hacia el exogrupo y más fuerte será la tendencia a tratar a los miembros del exogrupo como ítems indiferenciados de una categoría social. Esto quiere decir que los miembros del endogrupo se percibirán a sí mismos en términos de los estereotipos grupales y harán lo mismo respecto a los miembros del exogrupo. La teoría de la Categorización del Yo (Turner et al., 1987), que supone una extensión de la teoría de la Identidad Social, ha profundizado en estas consideraciones, estableciendo que el proceso fundamental que ocurre cuando determinada pertenencia categorial es prominente en una persona («saliente») es el de «despersonalización», es decir, la percepción de uno mismo en términos del estereotipo grupal. El concepto de saliencia o prominencia es situacional y depende de dos factores: la «accesibilidad» relativa de la categoría en el repertorio del perceptor y el «ajuste» entre la entrada y las especificaciones almacenadas en la categoría. Un determinante fundamental de la accesibilidad es la centralidad o importancia relativa de la pertenencia a un grupo particular para la definición del yo de un individuo (Oakes, 1990, pp. 179-181), así como el significado emocional o valorativo actual de una determinada categorización endogrupo-exogrupo (Tajfel, 1972). De esta manera, cuanto mayor es la identificación con una categoría y más importancia tiene esta identificación, más se verá la persona a sí misma en términos de los estereotipos que definen la categoría. En definitiva, según las teorías de la Identidad Social y de la Categorización del Yo, dos predicciones claras podrían hacerse respecto a identificación y estereotipia: 1) una situación intergru$\mathrm{pal}$, que haga saliente la identidad de género, llevará a esa persona a autopercibirse en términos estereotípicos asociados con su endogrupo de género; 2) cuanto mayor sea el grado de identificación de una persona con su endogrupo de género, mayor será la tendencia a autopercibirse en términos estereotípicos asociados con dicho endogrupo.

Además de estos dos factores, la autoestereotipia puede depender del status del grupo al que uno pertenece y de la valoración que tengan los atributos estereotípicos. Como han sugerido algunos autores (Cioldi, 1988), si la identidad es más saliente en los grupos de bajo status (esto es, en las mujeres), habría que esperar en éstas una mayor descripción según las características estereotípicas de su endogrupo de género. Asimismo, parece plausible suponer que la autoestereotipia será mayor en los atributos positivos que en los negativos, supuesta la tendencia a tener una autoimagen positiva.

A) Identificación con el endogrupo de género y autoestereotipia

Examinemos, en primer lugar, si hay una correlación positiva entre el grado de identificación con el endogrupo y la percepción de sí mismo en términos estereotípicos. En dos investigaciones (Moya, 1990, 1991), pudimos abordar es- 
ta cuestión, dado que los participantes contestaban de una parte a la escala de identificación grupal y de otra se autocalificaban sobre un serie de atributos característicos de cada grupo de género.

En la primera investigación (Moya, 1990), ni en el caso de los hombres ni en el de las mujeres aparecieron correlaciones significativas entre identificación y autoestereotipia (varones, $\mathrm{r}=.08$; mujeres, $\mathrm{r}=.11$ ). Tampoco aparecieron resultados significativos cuando se consideraron los diversos factores encontrados en la escala en vez de la puntuación global.

En la segunda investigación (Moya, 1991), en el caso de los varones la correlación entre identificación con el endogrupo de género y la autoadjudicación de características masculinas fue considerablemente alta $(r=.51 ; p<.01)$. En cambio, en las mujeres no resultó significativa la relación entre identificación y autoadjudicación de características femeninas $(\mathrm{r}=.16)$. En su caso fue incluso mayor la correlación de la identificación con la autoadjudicación de características masculinas $(r=.18)$. Si en vez de considerar la puntuación total en la escala de identificación grupal utilizamos las puntuaciones obtenidas en los tres factores encontrados en dicha escala (evaluativo-negativo, cognitivo y evaluativopositivo) encontramos en los varones sólo una relación significativa entre la autoadjudicación de atributos masculinos y el primer factor (de signo negativo: $\mathrm{r}=-.46, \mathrm{p}<.01)$ y el tercer factor $(\mathrm{r}=.37, \mathrm{p}<.05)$. En el caso de la mujer las correlaciones no fueron en ningún caso significativas (sólo se acercaba a la significatividad en el caso del tercer factor: $r=.16, p=.07$ ). Resulta, pues, interesante señalar la ausencia de relación significativa entre la identificación «cognitiva» con el endogrupo de género y la autoadjudicación de características estereotípicas. En cambio, sólo en el caso de los varones, cuanto más consciente era el sujeto de que su identidad de género tenía connotaciones negativas, más características típicas masculinas se autoadjudicaba, y viceversa, cuantas menos características masculinas se adjudicaba, menor era el sentimiento de que su identidad de género era negativa.

Los resultados de las dos investigaciones que acabamos de comentar no suministran, pues, apoyo para la noción de la TIS y de la Teoría de la Categorización del Yo, de que cuanto mayor es la identificación con el endogrupo, mayor será la autopercepción en términos característicos del grupo.

Sin embargo, otros autores sí han encontrado relación entre identidad y autoestereotipia. Gurin y Markus (1990) estudiaron el funcionamiento de la identidad de género (sólo en el caso de mujeres) concibiéndolo como un esquema del Yo. Los esquemas del Yo son generalizaciones cognitivas sobre el Yo en campos específicos, que llevan a organizar la experiencia de ese campo: modulan las expectativas, determinan a qué estímulos se les presta atención, guían el procesamiento de nueva información, ayudan a recuperar la información almacenada y constituyen la base para la realización de inferencias. En su estudio encontraron que las mujeres con una identidad de género más central en el concepto del Yo se adjudicaban a sí mismas más términos relacionados con el género, y lo hacían con mayor rapidez y seguridad. No obstante, hay que tener en cuenta que la medición que estos autores hicieron de la centralidad del género en la identidad es diferente de la que realizamos en nuestras investigaciones. Así, ellos preguntaban: «Indica la frecuencia con la que en tu vida cotidiana piensas sobre ser mujer y sobre lo que tienes en común con hombres y mujeres» (el formato de respuesta era: bastante, algo, un poco, nada en absoluto). 
B) Diferencias de status y autoestereotipia

Si tal y como hemos comentado anteriormente, la identidad de género tiene más importancia y es más saliente en las mujeres que en los hombres, esto debería verse reflejado en las concepciones que tanto unos como otras tienen de sí mismos. Es decir, las mujeres han de verse a sí mismas en los términos géneroestereotípicos de su endogrupo en mayor medida que los hombres. Los resultados de diversas investigaciones muestran que el apoyo no es desde luego unánime para esta suposición, como puede apreciarse en la siguiente tabla. En todas esas investigaciones los sujetos tuvieron que autocalificarse sobre una serie de atributos masculinos y femeninos. Tal y como hemos comentado, se considera que un individuo es masculino cuando puntúa por encima de la mediana en los ítems masculinos y por debajo en los femeninos; y que es femenino cuando puntúa por encima de la mediana en los ítems femeninos y por debajo en los masculinos. En tres investigaciones se confirma la anterior suposición: las mujeres se autocalifican más sobre los rasgos femeninos que los hombres sobre los masculinos; mientras que en otras tres investigaciones se desconfirma.

TABLA V

Porcentajes de individuos varones «masculinos» $y$ de mujeres «femeninas»

\begin{tabular}{lcc}
\hline Investigación & Varones masculinos & Mujeres femeninas \\
\hline Fernández (1982) & 28.26 & 29.42 \\
Páez et al. (1989) & 37 & 28 \\
Echebarría (1990) & 36.7 & 31.8 \\
Moya (1987) & 27.78 & 31.58 \\
Moya (1990) & 36.2 & 39.6 \\
Moya (1991) & 25 & 20.5 \\
\hline
\end{tabular}

C) Características de la $\cdot$ situación y autoestereotipia

Moya (1987) encontró que la autoestereotipia puede depender del tipo de situación en la que se encuentra el individuo (características propias de la situación, personas con las que se interactúa, etc.). Así, por ejemplo, el encuentro intersexual (intergrupal) disminuye en hombres y mujeres su autoclasificación en agresividad respecto al estereotipo grupal mientras que el encuentro con individuos del mismo sexo la aumenta. Hay, sin embargo, una interacción de esta tendencia con el sexo del contestante: en los hombres hay mucha mayor diferencia entre la adjudicación al grupo y la adjudicación a sí mismo (5.44 vs. 2.73) que en las mujeres ( 4.73 versus 3.56 ) (posiblemente porque en el caso de los varones existe una potente norma social que condena la violencia contra las mujeres). Asimismo, cuando se consideraba a todos los sujetos, éstos se veían a sí mismos como más asertivos que a su endogrupo de género (6.25 y 6.74, respectivamente). Sin embargo, en este caso también aparecía una interacción entre el sexo del sujeto y la situación por la que acababa de pasar. Los hombres se autopercibían como más asertivos que su grupo después de enfrentarse a dos mujeres (situación intergrupal) que después de hacerlo con otro hombre (6.67 y 5.71, respectivamente); en cambio, las mujeres se autopercibían con mayor asertividad después de enfrentarse a una mujer que después de hacerlo con dos hombres (6.75 vs. 5.87 ). 
En el mismo estudio se investigó si el incremento de la estereotipia sexual del Yo en hombres y mujeres que se encontraban en una situación intergrupal (en comparación con otros y otras que estaban en una situación interpersonal) se daba uniformemente en todos los ítems sexo-estereotípicos o sólo en aquellos que el individuo consideraba relevantes para esa situación. En una primera fase, los sujetos indicaron las características que manifestarían las personas de su propio sexo en una situación donde varias personas mantienen puntos de vista enfrentados. Se consideraron como estereotipos de género del grupo aquellos ítems que fueron elegidos como característicos por al menos el $75 \%$ de los sujetos. Estos estereotipos fueron: asertivo, dominante, enérgico, masculino, solemne (serio). Posteriormente los sujetos participaban en una tarea de discusión simulada y se clasificaban a sí mismos sobre las mismas características en las que antes habían calificado a la persona de su sexo.

Los resultados mostraron que en ninguno de los ítems considerado relevante por los individuos se producía un incremento de la estereotipia sexual del Yo respecto a la estereotipia sexual del grupo en la situación intergrupal en comparación con la situación interpersonal. Al contrario, en los ítems considerados relevantes se daba una disminución de la estereotipia del Yo respecto a la estereotipia grupal.

\section{TABLA VI}

Estereotipo del grupo y estereotipo del Yo en los ítems considerados relevantes para la situación.

\begin{tabular}{cccc}
\hline Item & Est. Grupo & Est. Yo & F \\
\hline Asertivo-a & 6.74 & 6.25 & $5.00^{*}$ \\
Dominante & 5.98 & 4.05 & $58.84^{* *}$ \\
Enérgico-a & 6.47 & 5.48 & $21.34^{* *}$ \\
Masculino-a & 5.19 & 4.26 & $14.40^{* *}$ \\
Solemne (serio-a) & 5.77 & 4.92 & $9.71^{\text {** }}$ \\
\hline
\end{tabular}

* $\mathrm{p}<0.05 ;{ }^{* *} \mathrm{p}<0.01$

Sin embargo, utilizando un procedimiento similar, Hogg y Turner (1987) sí encontraron un apoyo más claro para la teoría de la categorización del Yo. En su estudio variaron experimentalmente la saliencia de la categoría sexual de hombres y mujeres (haciéndolos interactuar bien en diadas del mismo sexo -baja saliencia - bien en parejas del mismo sexo que se enfrentaban a otra pareja del sexo opuesto - alta saliencia-) y diagnosticaron los efectos de tal variación sobre la estereotipia del Yo - descripción del Yo según estereotipos del propio sexo que el individuo tiene para una situación específica- y otras medidas evaluativas y conductuales. Los resultados mostraron, conforme predice concretamente la concepción de la saliencia de Oakes (1987), que en la conducta de alta saliencia los individuos se percibían a sí mismos más sexo-estereotipadamente y que la forma concreta de esa estereotipia y de otras medidas - autoestima, discriminación exogrupal, favoritismo endogrupal- dependía de la naturaleza de las relaciones entre los sexos en el grupo estudiado.

D) Valoración de los atributos estereotípicos

La teoría de la Categorización del Yo ha establecido que cuando una pertenencia grupal es saliente el individuo tiende a percibirse en función de los tér- 
minos estereotípicos de la categoría. La pregunta que surge es ¿en función de todos los estereotipos de la categoría? La teoría ha establecido que este proceso no se tiene por qué dar en todos los atributos estereotípicos sino en aquellos relevantes para la situación o en aquellos otros relacionados con la dimensión de comparación que se utiliza en una situación determinada. Por otra parte, las investigaciones de Codol (1975) y el conocido efecto «primus inter pares», indican que identidad de las personas no es un fiel calco o reflejo de su pertenencia grupal, sino que el individuo asume por una parte el estereotipo grupal, pero a la vez busca la diferenciación, lo que le lleva a autopercibirse como «estereotípico» pero «más estereotípico» que los otros miembros de su grupo.

Uno de los objetivos de la investigación de Moya (1987) era investigar si la estereotipia del Yo era mayor o menor que la estereotipia del grupo. Con tal propósito hicimos tres grupos de ítems. Tal y como se indicó, en una primera fase los sujetos dijeron si una serie de atributos eran o no característicos de un miembro de su endogrupo de género; además se les pidió a los sujetos que calificaran los mismos atributos según consideraran o no que eran «deseables» para la situación descrita (recuérdese: una discusión). Posteriormente, en otra fase, los sujetos se calificaron a sí mismos sobre los mismos atributos. El primer grupo de ítems lo forman lo que denominamos el estereotipo positivo: aquellos considerados característicos y deseables, o no característicos e indeseables. El segundo grupo son los ítems negativos: aquellos considerados característicos e indeseables, o no característicos y deseables. Por último, los ítems neutrales son aquellos que obtuvieron una puntuación intermedia (5) en alguna de las dos escalas («características» y «deseabilidad»).

El grado en el que un individuo estereotipa su Yo sobre un ítem específico es la diferencia entre la puntuación que da a dicho ítem en la primera fase del experimento (estereotipo de grupo) y la que le da en la segunda fase (estereotipo del Yo). Si el signo de esa diferencia es positivo, esto indica que el estereotipo del grupo es más extremo que el estereotipo del Yo, en cambio, si el signo es negativo, resulta que el estereotipo del Yo es más extremo que el estereotipo del propio grupo sexual. Estas puntuaciones diferenciales fueron promediadas entre todos los ítems pertenecientes a cada una de las tres categorías evaluativas - «tipo de ítem»-, hallándose así tres puntuaciones de estereotipia del Yo por sujeto: ítem tipo positivo, ítem tipo negativo e ítem tipo neutral.

Un análisis de varianza de tres vías (situación interpersonal/intergrupal $\mathrm{X}$ hombre/mujer $\mathrm{X}$ tipo de ítem positivo/neutral/negativo con medidas repetidas en el último factor), revelaba un efecto principal del tipo de ítem (F (2, 184) $=17.15, \mathrm{p}<.0000$ )

Todos los individuos tendían a mostrar una mayor estereotipia del Yo en

TABLA VII

Puntuaciones de los tipos de items.

\begin{tabular}{lrrrrr}
\hline Tipo de ítem & \multicolumn{2}{c}{ Interp. } & \multicolumn{2}{c}{ Intergr. } & \\
& Hombre & Mujer & Hombre & Mujer & Total \\
\hline Positivo & -0.13 & -0.08 & -0.04 & -0.04 & -0.08 \\
Neutro & 0.04 & 0.11 & 0.07 & 0.14 & 0.09 \\
Negativo & 0.15 & 0.18 & 0.23 & 0.38 & 0.24 \\
\hline
\end{tabular}


los ítems positivos que en los neutrales y que en los negativos: las puntuaciones de $-0.08,0.09$ y 0.24 difieren de forma significativa $(\mathrm{p}<0.01$ en la prueba de Newmanm-Keuls). No existieron efectos significativos ni del sexo de los sujetos ni de la condición experimental, así como tampoco aparecieron interacciones significativas. Estos resultados indican que los individuos tienden a describirse a sí mismos de forma menos extrema a como describen al grupo, pero sólo en los ítems negativos y neutrales y no en los positivos. Así, aunque parece confirmada la idea de que los sujetos no se aplican a sí mismos los estereotipos del grupo en toda su extensión, esta confirmación es parcial dado que en los ítems que el individuo considera como deseables el grado de estereotipia del Yo es mayor que el de estereotipia del grupo.

\section{DISCUSION}

En esta breve revisión hemos considerado algunas de las consecuencias cognitivas que tiene sobre la identidad de los individuos su pertenencia a una determinada categoría de género. Nuestro repaso no ha pretendido en absoluto ser exhaustivo. De hecho, los procesos o áreas que hemos tratado han sido seleccionados teniendo en cuenta que nuestra propia investigación tuviera algo que decir al respecto. Asimismo, la adopción - aunque no de forma estrictade la perspectiva teórica suministrada por la teoría de la Identidad Social, excluye la consideración de otras consecuencias cognitivas sobre la identidad, de índole más puramente cognitiva y estudiadas en Cognición Social, pero a la vez permite una articulación más rica de los diversos fenómenos entre sí y en relación con la realidad social.

Los resultados de las investigaciones que hemos presentado ilustran la existencia de parecidos y diferencias en las consecuencias cognitivas que tiene la pertenencia a la categoría hombre y mujer. Parecidos, en cuanto que ambos sexos tienden a tener un alto grado de identificación —en sus facetas cognitiva y evaluativa - con su grupo de pertenencia y tienden a autoadjudicarse, en líneas generales, los atributos estereotípicos asociados a tales grupos. Las diferencias surgen cuando se analizan los matices de la pertenencia categorial. En las mujeres parece existir un mayor sentido de grupalidad, que hace menos probable la utilización de estrategias individualistas para la obtención de identidad social positiva. En los hombres, en cambio, las alternativas grupales parecen menos pertinentes y se da la posibilidad de disminuir la vinculación cognitiva con el endogrupo de género si éste no proporciona una identidad positiva. También aparece cierta tendencia, tanto en hombres como en mujeres, a autoadjudicarse más los rasgos femeninos que los masculinos.

Por último, una serie de resultados sugieren el carácter dinámico y flexible de las consecuencias de pertenecer a una categoría de género. Los aspectos más estáticos de la pertenencia a estas categorías, como la fuerza de la identificación o el status de la categoría, no han parecido influir en la autopercepción conforme a las características estereotípicas del endogrupo. En cambio, sí han mostrado su influencia sobre estas percepciones ciertas características de la situación y de la persona con quien se interactúa, así como la evaluación que el individuo hace de los atributos. 


\section{Referencias}

Basow, S. A. (1980). Sex roles stereotypes: Traditions and altematives. Monterey, Calif., Brooks/Cole.

BEM, S. L. (1974). The measurement of psychological androgyny. Joumal of Consulting and Clinical Psychology, 42, 165-172.

Brown. R. J.; Condor, S.; Mathews, A.; Wade, G., y W WLLIAMs,J. A. (1986). Explaining intergroup differentiation in a industrial organization. Joumal of Occupational Psychology, 59, 273-286.

CoDor, J. P. (1975). On the so-called esuperior conformity of the self behaviour: twenty experimental investigations. European Joumal of Social Psychology, 5, 457-501.

Dron, K. L. (1975). Women's reactions to discrimination from members of the same or opposite sex. Joumal of Research in Personality, 9, 294-306.

Echebarrí, A. Gender social identity, values and cognitive processes. Manuscrito no publicado. Universidad del País Vasco.

FERNÁNDEZ,J. (1983). Nuevas perspectivas en la medida de la masculinidad y feminidad. Madrid. Editorial de la Universidad Complutense.

FRIEDL, E. (1975). Women and men. An anthropologist's view. Nueva York, Holt, Rinehart and Winston.

Gofrman, E. (1977). The arrangement between the sexes. Theory and Society, 4, 301-331.

Gurin, P., y Markus, H. (1989). Cognitive consequences of gender identity. En S. Skevington y D. Baker (eds.): The social identity of women. Londres, Sage. 152-172.

Heilbrun, A. B. (1981). Human sex-role behavior. Nueva York, Pergamon Press.

HoGg, M., y TURNER, J. C. (1987). Intergroup behaviour, self-stereotyping and the salience of social categories. British Joumal of Social Psychology, 26, 325-340.

Hurtig, M. C., y Pichevin, M. F. (1985). La variable sexe en psychologie: donné ou construct? Cabien de Psychologie Cognitive, 5 (2), 187-228.

Kunn, M. H. (1960). Attitudes by age, sex, and professional training. Sociological Quarterly, 44-55.

LoRENZI.Croldi, F. (1988). Individus dominants et groupes dominés. Images mascuilines et féminines. Grenoble, PUG.

McGurRe, W. J.; McGuire, C. V.; WINTON, W. (1979). Effects of household sex composition on the salience of one's gender in the spontaneous self-concept. Joumal of Experimental Social Psychology, 15 (1), 77-90.

Moya Morales, M. C. (1985). Identidad, roles y estereotipos de género. Revista de Psicología General y Aplicada, 42 (3).

Moya Morales, M. C. (1987). Percepción de episodios y estereotipia sexual. Tesis Doctoral, Servicio de Publicaciones, Universidad de Granada.

Moya Morales, M. C. (1990). Persistence and change of gender stereotypes. Comunicación presentada en el General Meeting de la European Association of Experimental Social Psychology. Budapest, junio.

Moya Morales, M. C. (1991). Social identity and gender stereotypes. Comunicación presentada en el Small Meeting about Changing Stereotypes. París, septiembre.

Moya Morales, M. C., y Pérez Meléndez, C. (1990). Nuevas perspectivas en el estudio de los estereotipos de género. En M. P. Valcárcel y J. L. Melia, G. (Comp.): Métodos y técnicas de investigación. Barcelona, PPU. Actas del II Congreso Nacional de Psicología Social, 49-59.

Moya Morales, M. C., y Gómez Berrocal, C. (1991). Psicología y género: un análisis de la profesión. Manuscrito no publicado. Madrid, Instituto de la Mujer.

Mulford, H. A., y Salusbury, W. W. (1964). Self-conceptions in a General Population. Sociological Quarterly, 5, 35-46.

OAKEs, P. J. (1990). La saliencia de las categorías sociales. En J. Turner et al.: Redescubrir el grupo social. Madrid, Morata.

Páez, D.; Torres, B., y Echebarría, A. (1990). Esquema de sí, representación social y estereotipo sexual. En Musitu, G. (Comp.): Procesos psicosociales básicos. Barcelona, PPU. Actas del II Congreso Nacional de Psicología Social 229-234.

SHERIF, G. W. (1982). Needed concepts in the study of Gender Identity. Psychology of Women Quarterly, 6, 375-389.

TAJFEL, H. (1972). La catégorisation sociale. En S. Moscovici (Ed.). Introduction à la psychologie sociale. París, Larousse.

TAJEL, H., y Turner, J. C. (1979). An integrative theory of intergroup conflict. En W. G. Austin y S. Worchel (Eds.): The Social Psychology of Intergroup Relations. Monterey, Calif.: Brooks-Cole.

TAJFE, H. (1984). Grupos bumanos y categorías sociales. Barcelona, Herder.

Turner. J. C.; Hogg, M. A.; OAkes, P. J.; Riecher, S. D., y Wheterell, M. (1990). Redescubrir el grupo social. Madrid, Morata. 